\title{
Relational groupoids and residuated lattices
}

\author{
Cosimo Guido \\ Department of Physics and Mathematics-University of Salento, Lecce, Italy \\ cosimo.guido@unisalento.it
}

Residuated structures are important lattice-ordered algebras both for mathematics and for logics; in particular, the development of lattice-valued mathematics and related non-classical logics is based on a multitude of lattice-ordered structures that suit for many-valued reasoning under uncertainty and vagueness. Extended-order algebras, introduced in [10] and further developed in [1, give an order-theoretical approach to a general description of the algebras of logics which goes along the line of an implication-based view.

In the metamathematical framework of classical and intuitionistic logics the algebraic structure of their semantics depends entirely on an order relation in the set of the truth values. In fact, the algebraic structure of both boolean and Heyting algebras (or, as one should prefer saying, boolean and Heyting lattices) is completely determined by the underlying order relation. Looking at non-classical logics, extended-order algebras have been introduced on the base of the following principle: "just like an order relation $\leq$ in a set $L$ determines completely the lattice structure of $L$, each of its extensions relative to a true value $T \in L$, e.g. any implication $\rightarrow: L \times L \rightarrow L$ such that for all $a, b \in L: a \leq b \Leftrightarrow a \rightarrow b=\top$, completely determines the richer lattice-ordered algebraic structure on $L$, to be used either in classical or in non-classical logics".

The implicative structure $(L, \rightarrow, T)$ so obtained has been called implicative algebra in the monograph of $\mathrm{H}$. Rasiowa [12, where it has been specialized to characterize either algebras of subsets (implication algebras) or algebras of open sets (positive implication algebras also called Hilbert algebras). Instead, the above described motivation has led to call $(L, \rightarrow, \top)$ weak extended-order algebra (w-eo algebra) in [10, 1]; there, additional conditions have been considered and discussed, including a weak, but important requirement that characterizes extended-order algebras (eo algebras), giving characterizations of several classes of residuated structures; in particular, it is seen that every integral residuated lattice is a symmetrical distributive and associative eo algebra.

It has to be noted that

- the associativity condition has not a fundamental conceptual role but only a technical strength;

- in a different way than in [7, the above mentioned approach to (non-necessarily associative) residuated lattices emphasizes the close connection the semantic of logics has with these structures, originally arising in a mathematical context [5];

- the restriction to the integral case only in the above described inclusion of residuated lattices in a subclass of $e o$ algebras is not so relevant from the logical point of view, since most algebras of logics, excluding rather weak substructural logics, are, in fact, integral residuated lattices;

- the above restriction to the integral case is, however, an unpleasant gap from the mathematical point of view. 
This proposed talk aims at closing this gap with a more general order-theoretic approach to residuated structures obtained by relaxing the requirements previously asked on the set $L$ of the truth values to get (weak) extended-order algebras, as follows

1. assume a preorder (not necessarily an order) on $L$;

2. assign a non-trivial set of values true, (not necessarily a singleton) in $L$.

The weakening given by the first requirement is not so relevant for our purpose, since one can easily reduce to the ordered case; so, we skip here that case. The second weakened requirement, instead, is crucial and allows a class of structures, which we have called weak implicative groupoids, consisting in a triple

$$
(L, \rightarrow, E)
$$

with $\rightarrow: L \times L \rightarrow L$ and $\emptyset \neq E \subsetneq L$, characterized by axioms which are equivalent to saying that the binary relation $\leq$ in $L$ defined, for all $a, b \in L$, by

$$
a \leq b \Leftrightarrow a \rightarrow b \in E
$$

is an order. Implicative groupoids are then considered adding antitonic condition on the first and isotonic condition on the second argument of $\rightarrow$. The ordered set $(L, \leq)$ is the natural context of the implicative groupoid $(L, \rightarrow, E)$. Conversely, for any ordered set $(L, \leq)$ and for any subset $\emptyset \neq E \subsetneq L$, every E-extension of $\leq$ to $L$, that is every $\rightarrow: L \times L \rightarrow L$ that satisfies the above equivalence, gives a weak implicative groupoid $(L, \rightarrow, E)$.

Note that any weak implicative groupoid determines uniquely its natural context; conversely, given a context (an ordered set $(L, \leq)$ ), each of the terms $\emptyset \neq E \subsetneq L$ and $\rightarrow: L \times L \rightarrow L$ in the equivalence $a \leq b \Leftrightarrow a \rightarrow b \in E$ determines, not uniquely but up to an equivalence, the other one.

The important point of view for our purposes has been to study, characterize and classify implicative groupoids $(L, \rightarrow, E)$ depending on which kinds of subsets are in the equivalence class of $E$ : interesting subsets, in this respect, are the order filters, in particular the principal ones, of $(L, \leq)$, or the possible top element, if any, of $L$, which are appropriate candidates for the set of values "true" in $L$. Suitable accommodation of conditions considered in [10, 1, for eo algebras, such as distributivity, associativity and a graduation of the symmetry condition allow to say, as one of the main results, that the right-distributive strongly symmetrical complete implicative groupoids are the non-associative generalization of complete residuated lattices. Wider classes of implicative groupoids that are reasonable generalizations of residuated lattices are also considered.

It is interesting to note that implicative groupoids and the more general relational groupoids (for which the natural context has no required condition to satisfy) are closely related to a quite popular type of relational systems. We mean systems which are triples $(X, Y, r)$ where $X, Y$ are sets and $r$ is a binary relation from $X$ to $Y$ either with no required condition (formal contexts of formal concept analysis [8], or Chu spaces [11], or interchange systems [4]) or with the requirement for $Y$ to be a frame and for $r$ to reduce to a frame map from $\mathrm{Y}$ to $\mathbf{2}=\{0,1\}$ in its second argument (topological systems [15]).

Many-valued generalizations have been considered as relational systems which are quadruples $(X, Y, L, \eta), \eta: X \times Y \rightarrow L$, with or without conditions on the sets $X, Y, L$ and on the $L$ relation $\eta$; the so called basis $L$, which in the above classical case is meant to be $\mathbf{2}$, may also vary in some class of possibly structured sets [4, 11, 14.

Suitable morphisms between possibly many-valued and possibly variable-basis relational systems may be considered, which are generalized, possibly variable-basis Galois connections, with 
a partial composition that may give a categorical arrangement of this matter [3] this way one may obtain either categories or semicategories and precategories, which are particular multiplicative graphs in the sense of Ehresmann [6].

Now, it is the case that we consider tied relational systems as quadruples

$$
(X, Y, L, \eta, E)
$$

where $X, Y, L$ are sets, $\eta: X \times Y \rightarrow L$ is an $L$-relation from $X$ to $Y$ and $E \subseteq L$ is a subset of $L$.

These are not simply generalizations of classical formal contexts but, rather, specific extensions of a uniquely determined natural context. In fact, each $(X, Y, L, \eta, E)$ determines a binary relation $r$ from $X$ to $Y$ by means of the equivalence, for all $x \in X, y \in Y$

$$
x r y \Longleftrightarrow \eta(x, y) \in E
$$

and hence a unique natural context $(X, Y, r)$ to which it is tied through the subset $E$ (which is determined up the equivalence $\equiv_{\eta}$ such that $E \equiv_{\eta} E^{\prime}$ if and only if $\left.\eta(x, y) \in E \Leftrightarrow \eta(x, y) \in E^{\prime}\right)$. Relational groupoids $(L, \eta, E)$ are the special instances, namely $(L, L, L, \eta, E)$, of tied relational systems and they are (pre)implicative groupoids if the relation of their natural context is a (pre)order.

This general framework, where we have located implicative groupoids, suggests the following remark concerning the morphisms between these, somehow untypical, algebraic structures.

Beyond the usual morphisms of universal algebra from $(L, \eta, E)$ to $\left(L, \eta, E^{\prime}\right)$, e.g. maps from $L$ to $L^{\prime}$ that satisfy suitable conditions with respect to the pairs $\left(\eta, \eta^{\prime}\right)$ and $\left(E, E^{\prime}\right)$ [9, 13, one could consider as morphisms the pairs of maps $(f, g)$, with $f: L \rightarrow L^{\prime}$ and $g: L^{\prime} \rightarrow L$ which are so called relative connections (in particular Galois connections if $L$ is a poset and each of $E$ and $E^{\prime}$ has an $\equiv_{\eta^{-}}$-equivalent upperset in $L$ ) (see [2, 3]). Suitable categories, semicategories and precategories of implicative groupoids could be considered and their properties and possible applications could be exploited (see [3]).

\section{References}

[1] M. E. Della Stella, C. Guido: Associativity, commutativity and symmetry in residuated structures, Order, 30(2) (2013), 363-401.

[2] M. E. Della Stella, C. Guido: The structure of many-valued relations I. Galois and Tarski connections, (work in progress).

[3] M. E. Della Stella, C. Guido, L. Ruzittu: The structure of many-valued relations III. Tied relational systems, (work in progress).

[4] J. T. Denniston, A. Melton, S. E. Rodabaugh: Formal concept analysis and lattice-valued Chu systems, Fuzzy Sets Syst., 216 (2013), 52-90.

[5] R.P. Dilworth, M. Ward: Residuated lattices, Trans. Amer. Math. Soc., 45 (1939), 335-354.

[6] C. Ehresmann: Catégories et structures, Dunod, Paris, (1965).

[7] N. Galatos, P. Jipsen, T. Kowalski, and H. Ono, Residuated lattices: An algebraic glimpse at substructural logics, Studies in Logic and The Foundations of Mathematics, vol. 151, Elsevier, 2007.

[8] B. Ganter, R. Wille: Formale Begriffsanalyse: Mathematishce Grundlagen, Springer Verlag, Berlin, Heidelberg, New York, (1999) (German 35 edition 1996/Englishedition).

[9] C. Guido, L. Ruzittu: Relational and implicative groupoids, (work in progress).

[10] C. Guido, P. Toto: Extended-order algebras, J. Appl. Log., 6(4) (2008), 609-626. 
[11] V. R. Pratt: Chu Spaces, Notes for School on Category Theory and Applications, University of Coimbra, Portugal, 1999.

[12] H. Rasiowa: An Algebraic Approach to Non-Classical Logics, Studies in Logics and the Foundations of Mathematics, 78, North-Holland, Amsterdam, 1974.

[13] S. A. Solovyov, Extended-order algebras as a generalization of posets, Demonstratio Mathematica, Vol. XLIV, No 3 (2011), 589-614.

[14] S. A. Solovyov: Lattice-valued topological systems as a framework for lattice-valued Formal Concept Analysis, (to appear in Journal of Mathematics).

[15] S. Vickers: Topology via Logic, Cambridge University Press, 1989. 